\title{
Complementarity Theory: Why Human Social Capacities Evolved to Require Cultural Complements
}

\author{
Alan Page Fiske \\ Department of Anthropology \\ University of California, Los Angeles
}

\begin{abstract}
This article introduces complementarity theory, which explains the psychology of cultural diversity as a product of evolved social proclivities that enable-and require-people to coordinate action in culture-specific ways. The theory presents evolutionary processes and psychological mechanisms that may account for the cultural variability of social coordination devices such as language, relational models, rituals, moral interpretations of misfortune, taboos, religion, marriage, and descent systems. Human fitness and well-being depend on social coordination characterized by complementarity among the participants' actions. This complementarity is based primarily on coordination devices derived from the conjunction of cultural paradigms and specific, highly structured, evolved proclivities. The proclivities have no adaptive value without the paradigms, and the paradigms have no meaning without the proclivities. They are coadapted to function together. Operating in conjunction with each other, proclivities and paradigms jointly define the generative structures for meaningful coordination of social interaction in each particular culture.
\end{abstract}

My aim in this article is to describe the natural selection of universal psychological mechanisms that result in cultural diversity. Complementarity theory posits that human social coordination is the product of structured psychological proclivities linked to corresponding cultural paradigms. Using innate expectations about the ways in which people encode and conduct social relations, children take primary responsibility in actively searching for the relevant cultural paradigms they need. Putting proclivities together with congruent paradigms, children learn to construct culture-specific coordination devices that enable them to interact in locally meaningful ways. The evolved proclivities and cultural paradigms are complementary: Both are necessary but neither is sufficient to permit complex social coordination. People cannot use either their socially transmitted paradigms or their evolved proclivities independently of each other. Combining them, humans devise and depend on diverse, flexible social adaptations.

I am very grateful for the support of National Institutes of Mental Health Grant MH 43857, which made this research possible. I thank Robert Boyd, Marilynn Brewer, Roy D'Andrade, Francisco Gil-White, and Nick Haslam for their extremely helpful comments on an earlier draft.

Requests for reprints should be sent to Alan Fiske, Department of Anthropology, University of California, Los Angeles, Box 951553, Los Angeles, CA 90095-1553. E-mail: afiske@ucla.edu.
These coordination systems make possible a second kind of complementarity: the complementarity of the respective actions of the participants. To cooperate, contest, or defect, it is extremely advantageous to know what other people want, judge, feel, think, and will do. Moreover, many kinds of complex mutually beneficial cooperation require this kind of complementarity: communication, exchange, division of labor, joint action, meeting at a known time and place, planning a schedule for the flow of work, conducting a complex ritual, making joint decisions and committing to collective behavior, acting in concert in relation to outsiders, or cooperating to sanction someone-any action whose outcome depends on cooperation based on shared understandings.

This complementarity in human interaction is usually made possible by participants' joint use of shared coordination devices to construct their own actions and to interpret others' actions. Moreover, unlike social insects and most eusocial mammals, humans are capable of generating an infinite number of social coordination devices based on each evolved social proclivity. They do this by combining an evolved social proclivity with different cultural paradigms. Furthermore, people can generate endless additional possibilities by combining multiple coordination devices. This generativity makes coordination devices uniquely versatile, permitting humans to use a limited num- 
ber of evolved proclivities to construct a multitude of varied, complex, yet flexible, rapidly mutable, and locally specialized social adaptations. This generativity results from the indeterminacy of the evolved proclivities that permits them to be combined with any congruent socially transmitted paradigms, but this indeterminacy has another consequence. It makes social coordination dependent on cultural paradigms: Virtually all human social coordination is organized with reference to culture-specific coordination devices. This is a primary source of cultural diversity.

However, although humans can construct innumerable devices for social coordination, these devices are not arbitrary or unconstrained. Structured psychological proclivities facilitate the invention, learning, use, social transmission, and transformation of congruent cultural paradigms. Conversely, humans slowly evolve structured psychological proclivities to learn to construct adaptive coordination devices using all the diverse paradigms that people transmit over the long run and across diverse cultures. These proclivities evolve much, much more slowly than cultures change and they cannot track cultural ephemera. However, there is strong selection-weighted according to the frequency, duration, and adaptive value of the set of previous cultural paradigms-for universal capacities to construct culture-specific coordination devices based on just the set of diverse paradigms that people transmit. In turn, these paradigms, although diverse and potentially infinite in number, are substantially constrained by existing proclivities. Nevertheless, there is latitude for humans to invent and learn new paradigms. Some of these paradigms may diffuse widely, endure over many generations, and permit people to construct fitness-enhancing coordination devices. When this occurs there is strong selection for psychological proclivities to evolve to facilitate reliable learning of these paradigms and versatile, proficient utilization of them to construct adaptive coordination devices. Hence, proclivities and paradigms are symbiotically coadapted to fit each other and to require each other.

\section{The Natural Selection of Generative Proclivities for Social Coordination}

Cultural coordination devices (CCDs) are cognitive, motivational mechanisms that permit complementarity of social action. By definition, a CCD is determined by a specialized evolved proclivity in conjunction with one or more complementary paradigms. In the simplest case, a CCD is the product of a proclivity conjoined with one congruent cultural paradigm, but most proclivities require linkage with multiple paradigms. Most complex CCDs represent combinations of proclivities together with their corresponding paradigms. CCDs include language, relational models, rituals, religion, many taboos, systems of punishment, moral interpretations of misfortune, and kinship systems for forming groups according to descent and marriage. Probably many other universal yet culturally diverse practices and institutions are CCDs, including shame, jealousy, joking relationships, and music (although the adaptive value of the latter two is uncertain). Humans are exceptional in the extent of their adaptive exploitation of CCDs, their extreme reliance on CCDs, and the extraordinary number, complexity, and intergroup variability of these CCDs. CCDs are usually based on a shared model or schema (cf. D'Andrade \& Strauss, 1992), or a syntax, paradigm, prototype, artifact, architecture, or landscape. Participants use this model to understand, anticipate, evaluate, facilitate, forestall, resist, or sanction other participants' actions and to construct their own actions. The paradigm or syntax-and the social coordination resulting from such an artifact-is often inaccessible to reflection and incapable of being articulated; people do not know how they coordinate, plan, construct their action, or interpret each other's action.

Most CCDs probably result from an evolutionary sequence that is described in detail later in this article. In outline, this sequence of adaptations begins when people invent a system for coordinating behavior (e.g., a form of communication, a system of exchange, a method for dividing labor, a ritual that defines social status, or a taboo that regulates sexuality). The invention is the product of an unusual, accidental experience, or results from individual or group creativity and learning. The innovation or modification in a previous system may be very small, but if it enhances fitness then natural selection will favor those who learn the invention most reliably and most rapidly and are most adept at using it. Natural selection will operate on ontogeny, attention, learning, primary reinforcers, emotions, memory, cognition, and motoric systems to strengthen all proclivities to consistently develop and effectively utilize this learned innovation. People are most likely to invent new cultural systems for coordinating behavior that take advantage of - that are facilitated by-the evolving psycho-social-developmental specializations. If these additional cultural coordination devices enhance fitness, are widely diffused, and persist for many generations, then natural selection will further expand the adaptive specializations so that they enable people to learn and use the new devices effectively as well. Thus, within the constraints of the psycho-social-developmental proclivities required to become proficient using a set of socially transmitted coordination devices, natural selection will favor the 
widest range of adaptive uses of these specialized adaptive proclivities.

In humans, natural selection has gone beyond facilitating individual learning and use of social coordination systems. People cannot use such systems alone. Hence, there is strong selection for the capacity to learn such systems from others-and to convey them to potential partners, especially close kin. There are tremendous advantages in learning all kinds of adaptive behaviors from others, although it is surprising that no other animal has a very versatile capacity to do this (see following). Such capacities may begin to evolve primarily because of their advantages for profiting from other's experiences and fortuitous discoveries, whether cumulated or not, and whether social or nonsocial: The aggregate knowledge and skills of the local population must always exceed what any individual can learn for itself in one lifetime. Or such capacities may have begun to evolve because of their advantages for learning local devices for coordinating social interaction. In either case, once people begin to evolve such capacities, they are tremendously advantageous both for nonsocial learning and for learning social coordination devices.

Thus, culture builds on psychological proclivities, constructing and reproducing coordination devices congruent with human psyches; natural selection assimilates in the genome the psychological potential to reliably learn and effectively utilize the full range of adaptive CCDs enduring in the population. Natural selection simultaneously fosters capacities to learn from others and cumulate socially transmitted knowledge and skills over successive generations. Indeed, natural selection favors the evolution of capacities to link these two sets of skills, resulting in the evolution of specialized capacities to learn local forms of basic systems for social coordination.

So at some point, Homo evolved to the point where people could learn social coordination devices from each other. Building on this, there was another evolutionary step, based on two factors. First, there are constraints on the number of adaptive specializations that can evolve for learning social coordination devices. Adaptive specializations for learning can require a lot of energetically expensive neurological capacity. They may be very slow to evolve, so that they lag far behind the creation of individually learned and socially constructed innovations. They may be difficult to isolate in their proper domains, interfering with each other so that domain-specific cognition is constrained and the evolution of domain-specific learning faculties is impeded. This means that it is functionally advantageous to develop learning proclivities that somehow combine two seemingly incompatible features: (a) the high degree of structure needed to enable people to reliably and quickly learn and proficiently utilize complex capacities and (b) great flexibility of functional applications. This means that if there are principles of learning and cognition that are powerful for solving diverse problems, they be will extremely advantageous.

A second factor contributes even more to this next evolutionary step. People have two complementary preadaptations: capacities to learn from others and capacities to learn some set of social coordination devices. There is a way to link these that enhances both and at the same time provides a powerful structure for learning functionally diverse capacities. This is to evolve the capacity to construct generative systems that define basic structure, relational principles, and operations that work well in diverse contexts but leave key determining paradigms or prototypes open to be learned from others. The great adaptive advantage of generative structures is their unique combination of versatility, learnability, and coordinative potential.

This is an evolutionary step with a great adaptive advantage for two reasons. First, the most valuable things to learn from other people are devices for getting along with them-for understanding their motives, emotions, evaluations, cognition, and probable actions. To construct effective complementary action (to cooperate, avoid, or contest others' action) one needs to discover the structures used to organize their social action. So capacities to learn from others will be especially advantageous if they evolve to include specializations for learning social coordination devices. This will permit complementarity in diverse, locally adapted, rapidly mutable social systems. Second, it is virtually impossible to inductively construct a model that predicts the actions of a complex organism without extensive prior knowledge of its motives and strategies; this prediction is feasible only if most of the structure of that action is known a priori, so that there are only a few points of indeterminacy. To obtain the great benefits of coordinating social action-including the benefits of strategies for coordinating either conflict or cooperation-people must evolve psychological structures that they use jointly to construct action and to interpret it. For example, I can generally understand your action and know that you will generally understand mine if we both use the same device for constructing our respective actions and for interpreting each other's actions. It is possible to do this and still construct innumerable coordination devices if much of the structure of those devices is innately shared but certain paradigms are left open for learning and mutual social determination-if natural selection can construct mechanisms for this learning and joint determination of paradigms. A plausible mechanism is sketched in the section on ontogenetic externalization. 


\section{The Functional Interdependence of Evolved Proclivities and Cultural Paradigms}

The key concept in complementarity theory is that people have highly structured, evolved (predominately universal), attentional, motivational, cognitive, and developmental proclivities for discerning congruent cultural paradigms and using them to construct and utilize local cultural coordination devices. I have coined the term mod for such a proclivity. (The term is based on the Indo European root for mediate, mold, accommodate, modify, mode, model, and the Latin modus-measure, standard, size, limit, manner, harmony, melody). Mods have three defining features. First, they are innate proclivities for learning and performing structured behaviors, and in this respect they are more or less similar to (and build on) a number of important concepts in the existing literature: adaptive specializations for learning (Gallistel, 1995; Rozin, 1976), learning programs (Pulliam \& Dunford, 1980), transmission coefficients for cultural selection of traits (Cavalli-Sforza \& Feldman, 1981), epigenetic rules that affect usage bias curves for culturgens (Lumsden \& Wilson, 1981), direct biases or evolved predispositions guiding the adoption of cultural practices (Boyd \& Richerson, 1985, 1989), functional modules (Fodor, 1983; Hirschfeld \& Gelman, 1994; Tooby \& Cosmides, 1992), or primary values (Durham, 1991).

Second, mods are incomplete without a congruent socially transmitted complement. That is, a mod is insufficient for structuring action until it is linked with appropriate cultural paradigms that specify how, when, and where it operates. Third, a mod in conjunction with congruent cultural paradigms constitutes a cultural coordinating device that permits complementarity of action, cognition, emotions, motives, and evaluations. In short, a mod is a structured proclivity for learning and action that, when conjoined with the necessary type of cultural complement, permits culture-specific forms of social coordination based on complementarity of action. These last two features differentiate mods from the concepts cited in the preceding paragraph: Mods are not simply tendencies to learn selectively. Mods are adaptations for coordinating social interaction with reference to local cultural paradigms or prototypes. Mods are proclivities to learn from other people how to get along with them.

The theory is called complementarity theory for two reasons. First, it posits close complementarity between mods and socially transmitted cultural paradigms. This complementarity is hypothesized to consist of close historically causal linkages between mods and corresponding cultural paradigms that have coevolved. A mod can be adaptively expressed in the behavioral phenotype only in conjunction with one or more congruent cultural paradigms.

A second, closely linked dimension of complementarity is both cause and effect of the first type of complementarity. Humans create extraordinarily complex and diverse social practices, relationships, groups, and institutions in which the action of each person only makes sense in terms of the actions of the other participants and observers. Participants' actions are often mutually presupposing and mutually completing: The fulfillment of the participants' intentions depends on the congruence of others' actions. This is essential for large-scale cooperation, especially when it goes beyond immediate face-to-face responses. This social complementarity results from joint usage of the coordination devices that people construct by joining a mod with a congruent cultural paradigm.

Research on the coevolution of human psyches and cultures shows that social inventions form an important aspect of the environment that shapes natural selection and evolved psyches form an important component of the selective environment for cultural transmission (Boyd \& Richerson, 1985; Caporael, 1997; Durham, 1991; Lumsden \& Wilson, 1981; Pulliam \& Dunford, 1980; Rozin, 1982). The human invention, retention, use, transformation, acquisition and interest in cultural practices, artifacts, symbols, and institutions depends on the compatibility of these cultural paradigms with mods. Lumsden and Wilson (1981) described this as the bias curves that represent the translation of epigenetic rules into ethnographic curves. Boyd and Richerson (1985) called this direct bias in the adoption of cultural variants. Atran (1998) made a similar point with regard to adaptive habits of mind that universally shape folk biological classification, and Sperber (1996) took an analogous perspective when he described culture as infectious ideas to which human psyches are susceptible (cf. Boyer, 1994). Complementarity theory goes beyond this to posit that human social coordination is based primarily on cultural coordination devices that people construct by putting mods together with corresponding cultural paradigms. Mods cannot function and are not adaptive until conjoined with congruent socially transmitted complements. Conversely, mods permit humans to learn and to use cultural paradigms to construct culture-specific coordination devices. Thus, complementarity theory posits that natural selection, ontogeny, psychology, social organization, and culture are mutually potentiating and mutually determining. To delineate the theory there are four basic concepts that need to be considered more fully: (a) cultural coordination devices, (b) mods, (c) preos, and (d) ontogenetic externalization. 


\section{Concepts}

\section{Cultural Coordination Device (CCD)}

In essence, $\mathrm{CCD}$ are the core of the phenomena the theory attempts to explain. CCDs exhibit both universal fundamental principles and diversity across cultures. This is evident in relational models, rituals, religion, taboos, systems of punishment, moral interpretations of misfortune, kinship and marriage systems, perhaps language, and probably many other practices and institutions.

Relational models are CCDs (Fiske, 1991, 1998: Fiske \& Haslam, 1996). Relational models are universal structures that people implement in culture-specific ways to organize mutually intelligible social interactions: making group decisions or moral judgments; organizing labor; exchanging, distributing, and contributing; using objects, time, or space to mediate social interaction; or coordinating conflict and aggression. There are four relational models that people in all cultures apparently use to cognize, construct, comprehend, coordinate, and evaluate most social interactions, groups, and institutions. These models are communal sharing, authority ranking, equality matching, and market pricing (Fiske, 1992). Each model defines a set of relations and operations that are socially meaningful. For example, in aspects of an interaction organized with reference to communal sharing, people divide the world into social categories that are equivalence classes. With regard to the given aspect of the interaction, people within each category are not differentiated but each category is entirely distinct. For example, when people organize labor according to communal sharing, the task is a joint, shared responsibility of the participants and it does not matter which of them does what. If people consume something (e.g., food) according to communal sharing, then they just dig in and help themselves without being concerned about the amounts each person consumes: "What is mine is yours." Other models define additional relations and operations. For example, if people work according to equality matching, they divide the work up, being concerned about whether each person's tasks are equal, or they take turns at each task. People consuming a resource in an equality matching mode match their shares so they correspond one to one; if one person gets more than another, all participants know what they need to do to make things even.

The mod that is fundamental to each relational model specifies the meaningful kinds of relations and operations but is incomplete: The mod does not delineate when, how, or with whom to implement it. To use a model to coordinate a social interaction, participants need shared cultural paradigms, precedents, or principles that indicate some way to use the abstract structure. In communal sharing, for example, there must be a process that assigns persons to social categories: by descent, by contract, by residence, by gender, or whatever. To implement equality matching, people need cultural practices that define what tasks to organize this way, what counts as a turn, and what operations to use to compare shares. For example, to operationalize equality matching as a form of exchange, the participants must have a shared understanding of what kinds of entities can be exchanged in this manner, what constitutes an offer and acceptance of something proffered, what constitutes a return of the "same" thing or value, what is the proper interval between receiving and giving a return, permissible limits to imbalance, and so forth. Without these shared understandings to coordinate interaction, it is impossible to generate the complementarity of action necessary for effective social relationships. If a Fulani man gives you a cow, even if you guess correctly that the framework is equality matching, there is a lot more you need to know to figure out how to respond.

Language appears to be a CCD. Most linguists and psychologists have long accepted Chomsky's $(1959,1988)$ argument that a child could not learn a language without prior knowledge of a universal grammar and innately structured language acquisition device. No one speaks universal grammar (UG) and, according to the theory, it cannot be used to communicate until a finite number of parameters are set to specific values; to specify a particular language, each parameter must be set to one of a small number of possibilities (perhaps two). If this theory is correct, then UG together with the child's language acquisition device is a mod. However, the theory of UG and parameter setting remains controversial (e.g., Deacon, 1998; Elman, Bates, \& Johnson, 1996; Tomasello, 1998). Furthermore, there is no stable consensus on how to characterize universal grammar, its parameters, or the language acquisition device; and after 40 years there is little or no empirical evidence that directly supports the existence of a UG. Nevertheless, humans seem to have species-specific capacities that presumably evolved because of the enormous adaptive advantage conferred by linguistic abilities.

Another CCD is ritual. People engage in rituals for certain well-delineated purposes: to transform social status, mark life transitions, constitute social relationships and group membership, to cure ills, to redress wrongdoing or protect against misfortune. Rituals all over the world are composed primarily from a very limited, well-specified, contextually distinctive repertoire of actions, ideas, and emotions (Dulaney \& Fiske, 1994; Fiske \& Haslam, 1997). Among the most com- 
mon of these are (a) repetition of the same action, word, song, or sound; (b) a focus on special numbers and colors; (c) concerns about pollution and purity and consequent washing or other purification; (d) contact avoidance; (e) special ways of touching; (f) fears about immanent, serious sanctions for rule violations; (g) a focus on boundaries and thresholds; and (h) symmetrical arrays and other precise spatial patterns. Yet, the rituals that people compose from these elements are infinitely varied and unique to each culture. Evidently the psyche resonates with certain ideas and actions, providing a repertoire for composing rituals. The specific content, the arrangement of components, and the precise uses of this repertoire are open and must be culturally determined.

Another major CCD consists of beliefs, fears, and practices related to the moral interpretation of misfortune. People everywhere commonly assume that when something very bad happens, someone must have done something bad. That is, in every culture, people attribute many or all deaths and much suffering to wrongdoing: by the victim or against the victim. For example, the Tumbuka of northern Malawi attribute severe and persistent coughs such as tuberculosis to adultery-adultery committed by a parent, if the patient is a child (personal observations). In the biblical story of Job, Job's neighbors assume that his misfortunes and the suffering of his family must be due to his having angered God. Among the Moose (pronounced $\mathrm{MOH}$-say) of Burkina Faso, people consult diviners whenever anyone falls ill and they interview the deceased, seeking to determine the moral meaning of the illness or death. They assume that such suffering could result from violation of a taboo (violation committed by the sufferer or by a kinsperson or spouse), witchcraft or sorcery against the victim, or the anger of the ancestors or other beings who have not received their due sacrifices (personal observations). As in every other culture, these attributions about misfortune focus overwhelmingly on a search for some kind of transgression of a social relationship with another person, spirit, ancestor, or god.

People seek this kind of explanation even if they are satisfied that they fully understand the biomechanical or material causes of their suffering: They want something more. What they seek is not just any explanation that would give them some sense of predictability and meaning: In every culture people focus predominately on social transgressions. Nonsocial interpretations are always less prevalent and less emotionally satisfying. (People may also use astrological or numerological accounts, but moral explanations are almost always much more important.) In a great many cultures after a major misfortune, people attempt to redress the moral disequilibrium by making some kind of sacrifice that consists of a gesture of obeisance to gods, ancestors, or other superior beings and an attempt to renew solidarity with them. This basic approach to making sense of misfortune is universal, yet cultures vary greatly in their attribution of suffering to specific transgressions and relationships with specific types of beings, as well as the oracular processes they use to determine who has committed what transgression. To know what to do when you fall ill among the Azande, you have to know the culture.

Sex and food taboos with a certain characteristic structure represent another CCD. There is evidence that in every culture where there are important, institutionalized communal sharing relationships, these relationships entail a strong taboo concerning food, sex, or both (Fiske, 2000). Participation in the communal sharing relationship is contingent on observing the taboos: Observance is partially constitutive of the relationship. For Western readers, the most obvious examples are the incest taboos inherent in familial relationships, and the celibacy and food proscriptions entailed by joining a monastery or marrying God (becoming a nun). South Asians will immediately think of their caste rules. For Africans, totemic taboos associated with clans and protective associations, and the sex and food taboos associated with blood brotherhood will spring to mind. Readers from the Mediterranean and Central America will recognize their strongest taboos: against sexual relationships with the spouse of a trusted compadre. Traditional Melanesians will reflect on the numerous intense food and sex taboos incumbent on initiated males in a communal long house. There are innumerable other examples. All share certain remarkable structural features. The most notable feature is that the taboos proscribe a combination of three or more relationships that are innocuous in isolation from each other. It is taboo to engage in one or more particular triadic configuration of these relationships. For example, Americans value romantic sexual relationships, marriage, and parenthood, but it is taboo to combine these three relationships-it is not good to have a sexual relationship with the daughter of your spouse. Despite these common structural features, the examples demonstrate the diversity of the particular communal sharing relationships and specific food and sex interactions that are taboo in different cultures. The universality of taboos with this distinctive structure reflects the underlying mod; the necessity for completing these mods with specific cultural paradigms is evident in cultural specificity of the constellations that are taboo.

Religion, another CCD, consists partly of ritual, moral interpretation of misfortune, and taboos. Religion also involves two other elements: some kind of deferential (authority ranking) relationship with one 
or more immaterial superior beings and a desire to develop a relationship of solidarity or oneness (communal sharing) with the immaterial being or beings, with some ultimate essence, with the congregation as a whole, or all of these. Needless to say, these features are present in virtually all cultures, yet the world's religions have many distinctive features (cf. Boyer, 1994).

There appear to be many other CCDs; in any event, researchers need to further analyze these CCDs and search for more.

\section{Mod}

A mod is an innate proclivity to coordinate social interaction in a universally structured yet culturally organized way. It is an evolved but incomplete attentional-cognitive-learning-motivational system for organizing social interaction that cannot function without a congruent cultural complement. Many (although not necessarily all) of the following characteristic features should be present before something is called a mod:

- A quasi-universal socially transmitted coordinative practice (CCD) with a distinctive, consistent structure; coupled with a generative capacity to construct indefinitely many culture-specific systems, group- or dyadspecific practices, and unique interactive events.

- Virtually universal adult social competence for participating effectively in a given type of CCD despite vast differences in general intelligence and individual experience.

- A highly structured learning mechanism that results in competence that would be difficult to learn on the basis of individual experience without strong innate expectations (Bayesian prior hypotheses), yet that works very reliably with a distinctive kind of highly variable and imperfect cultural input.

- A sensitive period during maturation in which competence in the CCD can be acquired and retained much more readily, fully, and precisely than at any subsequent time.

- Perceptual capacities, attentional filters, and motivational effects tuned to a distinctive semiotic modality (sign system, communicative channel, or social medium) for learning, marking, and constituting a particular type of CCD (e.g., creating communal sharing relationships by eating and drinking together).

- Well-structured enactive and performative capacities that are partially preformed and emerge with minimal practice; performative capacities are linked to selective perceptual capacities, attentional filters, and constitutive modality.

- Focused, directive motives for acquiring and participating in a specific type of coordinative structure, sometimes overriding simple reinforcers, distraction, and fatigue.

- Active search initiated by the child, who experiments and innovates in ways not readily induced from the input stimuli and who makes constructive mistakes that could not result from simple associative induction.

- Signs of the unrealized potential of the mod in cultures in which the competence is not valued or not manifestly elaborated on the surface. For example, children may inventively initiate utilization of the $\mathrm{CCD}$, which may subsequently be lost, channeled into something else, or suppressed. Perhaps repression is evident: In dreams or fantasy, adults may exhibit covert signs of the cultural loss or mental suppression of the mod.

- Distinctive combinatorial properties: characteristic tendencies to combine to form CCDs with certain other mods, to remain independent of other mods, or to oppose certain other mods (cf. the related concept of cultural selection according to secondary values; Durham, 1991).

- Crucial role in coordinating human sociality: Important kinds of social complementarity depend on it.

- Significant adaptive advantage resulting from plausible process of natural selection.

- Homologous (albeit much simpler) social behavior in great apes and possibly other highly social primates and mammals.

- Functional neurological modularity, indicated by capacity to solve relevant problems in parallel (without interference) with other cognitive tasks, distinctive memory systems, problem-solving procedures, or types of errors.

- (?) Anatomical localization, as evinced by imaging, by phylogenetic anatomical comparisons, and by specific dysfunctions resulting from delimited lesions. ${ }^{1}$

Note that many evolved, function-specific mental modules are not mods because their function does not depend on being conjoined with any particular type of congruent cultural complement. For example, neither the adaptive specializations for recognizing faces, nor for distinguishing material objects and interpreting motions, are mods. Nor is cultural variation in some domain of behavior indicative of a mod. By definition there is no mod unless the proclivity re-

\footnotetext{
${ }^{1}$ There are somewhat modular neurocultural systems such as language that are clearly based on mods. However, some neurological modules, such as reflex arcs or facial recognition (if that is a module), are not mods because they do not presuppose culture or depend on any culture to be fully functional. Conversely, it is also quite possible that there are mods that cannot be isolated in any region of the brain, and that do not correspond with any discrete neurological circuits. For example, some mods may have evolved as interactions or co-adaptations of multiple neurological systems, each of which also has other, only indirectly related functions.
} 
quires a cultural complement to function. Hence, although food and sexual attraction are susceptible to cultural influence, aversion to chili peppers (Rozin, 1982) and male attraction to youthful women with regular features (Buss, 1994) are not mods. However, the proclivity for shame probably is a mod because the factors eliciting shame and the practices for dealing with it must be provided by the culture.

One of the central hypotheses of complementarity theory is that these 15 features are systematically related and hence should be highly correlated, but it does not follow that they should always occur together. Mod is a polythetic concept. Much further research will be needed to determine the extent to which these features do co-occur. Due to the fact that cultural reproduction and diffusion, natural selection, cognition, development, and social relations are dynamic processes acting on each other in a continuously shifting balance that never reaches equilibrium, it seems likely that "modity" will be a matter of degree: There may be intermediate types of adaptive specializations that substantially shape some aspects of social interaction and are amenable to substantial cultural molding, amplification, or inhibition, yet do not require one particular, distinctive type of cultural complement to operate. There is a need to reanalyze from this perspective infant and parental attachment, male proprietary sexual jealousy, and aversion to sexual relations with persons who were intimate coresidents during childhood.

The existence of a mod does not invariably result in the construction of a corresponding CCD and the fact that a certain type of CCD is not universal does not imply that there is no corresponding mod. Bear in mind that mods may push in diverse directions, may counteract each other, and can interact to produce cultural coordination devices that are not additive sums of the proclivities that potentiate them. There are cultures with little or no hierarchy, groups without religion, and individuals sexually attracted to animals. However, hypothesizing a mod requires explaining cases where it is not directly expressed.

\section{Preo}

To implement a mod to construct meaningful, coordinated interaction, people need shared indicators for how, when, and with whom to use the mod. We call such an indicator a preo because preos take the form of precedents, prototypes, paragons, precepts, propositions, practices, and paradigms. (Up to this point in the text, the term paradigm has been used instead of preo to avoid defining a new term before it was necessary.) A preo is the complement to a mod, completing the mod by specifying how to implement it as a particular CCD. Like a key or catalyst, a preo has to correspond to the nature of the indeterminacy of the mod. For example, in a given context the implementation of authority ranking may be modeled on representations of the comportment of a cultural paragon: a hero, media star, mythical figure, or god. Balancing shares in equality matching may be based on a process - a concrete operation that determines what counts as equal (e.g., weighing amounts in a pan balance, counting out items or aligning them in one-to-one arrays, or having the person who divides the shares take the share left over after others take their pick). According to the Chomskian view, the setting of parameters turns universal grammar into a particular syntax. People often construct and legitimate ritual performances to perpetuate their identity-defining traditions by reproducing "what we have always done." Other rituals are defined as reenactments of a prototypical event.

Complementarity theory suggests that cultural reproduction, diffusion, and acquisition of CCDs consist of the transmission of preos; likewise, cultural transformation consists of modification of preos. Hence, it is not a religion that is transmitted but only the paragons and practices that orient the relevant mods and permit children to shape their dispositions to be congruent with the particular local practices. Children do not "learn" the sexual disgust, disinterest, and fear that is the core of incest taboos: They learn whom to avoid and some ideas about what kind of misfortune may befall transgressors.

\section{Ontogenetic Externalization}

Complementarity theory suggests that children become cultural in large part at their own initiative. Children learn their culture because they there are insatiably social: They want to communicate and participate in relationships and daily activities (see Trevarthen, 1988). To coordinate with the people around them, children (or immigrants or anthropologists) need to determine the preos that will permit them to transform their mods into the CCDs that people in their community use to construct, comprehend, and evaluate actions. Rather than describing norms as being internalized by children who utilize social relationships as means to asocial ends, complementarity theory describes inherently sociable children who externalize their mods to connect with their families and communities. Children actively (though unreflectively) attempt to use their mods to interpret and to evaluate what goes on around them and construct relationships with those they encounter. Guessing and attempting to discern the correct preos to complete their mods, children try out various implementations 
of their mods in the hopes of coordinating interaction successfully. They initiate interactions, seeking to discover the preos that will complete their mods and satisfy their social appetites.

There are infinitely many preos for implementing any mod; hence, there are infinitely many possible CCDs. This creates a considerable problem for the child, the immigrant, or the anthropologist in learning a culture. Indeed, the problem appears as though it may be insoluble. How could a naïve person recognize the preos for implementing any mod? One seemingly insuperable difficulty is that the naif has to guess which mod is operative before discovering which preo is being used to implement it. That is, the naïve individual has to decipher combinations of mods and preos without initially knowing what either of them is. How do we do this?

Mods, preos, and CCDs generally are not readily accessible to reflective understanding or articulate expression. Who can explain the phonetic system, let alone the syntax, of their own language? Who can describe the operations and relations defined in everyday market pricing interactions such as buying groceries? (Who can describe the distributive law that partially defines an ordered field, or the necessary Archimedian property?) People do not learn most CCDs by instruction; for example, no one explains most of what you know about how to interpret misfortune. If someone tries to explain why it is wrong to have sex with one's mother, they cannot really explain why it just feels disgusting or awful. Adults do not have the capacity to teach CCDs and in most cultures adults and older children provide very little or no instruction to children (Fiske, 1999; Lancy, 1996). However, children do not need instruction to become competent in most aspects of most cultures.

Children learn most of what they need to know by observation, imitation, and incremental participation (Fiske, 1999). In general, children establish their first relationships by imitating each other (Eckerman \& Didow, 1996) and become proficient cultural participants by observing and then gradually participating more competently in more of the everyday practices of older children and adults (Greenfield, Maynard, Boehm, \& Yut-Schmidtling, in press; Lancy, 1996; Lave \& Wenger, 1991; Rogoff, Baker-Sennett, Lacasa, \& Goldsmith, 1995). Even technical skills are typically learned primarily by observation and apprenticeship involving guided participation or legitimate peripheral participation. Imitation may seem simple, but in fact only humans have evolved very sophisticated capacities to observe others, understand the functional relations of means to ends, and imitate the purposeful sequence (Nagel, Olguin, \& Tomasello, 1993; Tomasello \& Call, 1997; Tomasello, Kruger, \& Ratner, 1993; but see Whiten \& Custance, 1996).
We know very little about the cognitive processes involved in imitation, and almost nothing about the hypothesized specializations for learning preos. This crucial aspect of complementarity theory remains to be worked out - as it does for all other theories of evolution, psychology, culture, and development. The hypothesis, based on ethnographic evidence, is that children and adults arriving in novel cultures have strongly focused prior expectations about the ways in which people signal the social actions based on particular proclivities (see Fiske, 1991, pp. 148-149, 203-207). Apparently, mods are conjoined with focused prior expectations about the specific modality of the corresponding preos and default domains in which they are most likely to be utilized. For example, children expect communal sharing relationships to be constituted in part by breast-feeding and by commensality (eating and drinking the same substances together from the same vessels). Although this cue is not invariably correct, in every culture consistent commensality-especially ceremonially highlighted sharing of food, drink, and other comestibles-indexes all or most of the important communal sharing relationships (see Sahlins, 1972, pp. 215-219). Furthermore, suppose that children expect communal sharing also to be indexed by birth, by sleeping in the same space, by coresidence, and by similarity in appearance, dress, and adornment. These expectations do not fully determine or absolutely limit the possible preos for communal sharing, but if some of the local mod-preo linkages do correspond to a priori expectations they make it possible for naifs to bootstrap themselves enough to learn the other, culture-specific mod-preo combinations for which they are not innately prepared.

In contrast, children expect that when people are marking hierarchical positions, superior people will be higher up, assume more upright postures, assume positions in front, have the right to act first or go ahead, be accorded more personal space, wear accoutrements that make them appear bigger and higher, be accorded bigger shares and bigger items, be treated as if they were plural (e.g., royal we and respectful vous), be regarded as if they were more powerful, stronger, and more forceful, and be entitled to speak louder. Although not all of these expectations will fit every hierarchy in every culture, many of them will fit most of the important hierarchies in every culture. In any culture, one can reliably bootstrap the fundamental hierarchies, discovering who occupies what ranks, and when and where and how authority ranking operates with respect to what social domains. From these initial clues the naif can discover unique cultural cues such as the chiefly prerogative to construct rectangular houses, place ostrich eggs at the roof corners, and be greeted with an expression that means, "May your place be 
beautiful" (traditional norms of the Moose of Burkina Faso).

If people signal the paradigms and prototypes for different proclivities in distinctive modalities (if people conduct different CCDs in different interactive media and use these media in predictable ways) this enormously simplifies the problem of discerning the locally shared paradigms. However, we still need to learn much more about learning.

\section{Natural Selection for Ontogenetic Strategies}

Why do humans depend so extensively on learning coordination devices from other humans? To answer this question one needs to consider a broader issue, the evolution of ontogeny. When does natural selection result in genotypically fixed behaviors or plasticity, and how does it produce any given learning strategy?

\section{Baldwinian Adaptation}

A century ago Baldwin (1896), Morgan (1896), and Osborn (1896a, 1896b) simultaneously proposed a theory connecting learning to natural selection. What follows is a modern restatement of their idea. Within any population there is genotypic variation affecting the behaviors that different organisms exhibit in given conditions. When a population encounters new conditions, there will inevitably be differences among individuals in the behaviors they display. A behavioral adaptation can originate as a learned behavior that provides the learners with a selective advantage compared to nonlearners. Animals more likely to learn the adaptation reliably and rapidly have greater fitness than animals less prone to learn it. If the environmental conditions are relatively uniform and constant over a number of generations, the likelihood of any animal displaying the adaptive behavior increases. Within the often significant constraints imposed by the developmental and neurological costs of canalization, natural selection will produce animals that display adaptive traits most rapidly and reliably under the widest variety of experiential conditions. The behavioral adaptation can become fixed so that the animals display it even prior to any relevant experience. The behavior that was originally learned has become assimilated into the genome.

Baldwinian adaptation enables organisms to evolve complex combinations of interdependent traits that only enhance fitness in conjunction with each other. Furthermore, as Hinton and Nowlan (1987) first simulated, and Mayley (1997) and others further demonstrated theoretically, Baldwinian processes make it possible to evolve complex adaptations that are only functional when perfected: Maladaptive chasms between fitness peaks in the adaptive landscape may be bridged by plasticity in learning that permits animals to discover these complex adaptations without persistently exhibiting maladaptive intermediate phenotypes (cf. Laland, Richerson, \& Boyd, 1993).

Waddington (1975) confirmed empirically that such a process can occur. In a series of experiments on Drosophila beginning in 1956, Waddington artificially selected animals that acquired certain anatomical traits under specified environmental conditions. He showed that after a number of generations many of these traits appeared under a wider range of conditions: the Drosophila developed to display the trait reliably in the absence of the conditions that were originally necessary to induce its ontogenetic acquisition. Waddington called this process of narrowing the range of variability and increasing the probability of a given adaptive endpoint of ontogenetic development "canalization." Waddington thus proved that under strong selection it was possible for a trait acquired developmentally in earlier generations to become genetically assimilated. Although Waddington was aware of Baldwin (1896) and Morgan (1896), he analyzed this process only with regard to anatomical traits and did not explore learning or behavior in any detail.

Baldwin (1896), Osborn (1896a, 1896b), and Morgan (1896) assumed that learning of adaptive behavior would inevitably pave the way for assimilation that fixed the behavior more rigidly in the genotype. The crucial point that they ignored was that under many conditions the adaptive advantages of learning would lead to more effective and more specialized learning, rather than a genetically fixed action pattern. The equilibrium point of selection is somewhere in between random unstructured learning, at one extreme, and behavior rigidly canalized by the genotype, at the other extreme. That is, the function that maps experience onto behavior is itself an adaptation. Natural selection can move ontogenetic strategies in either direction and, at the plastic end of the continuum, can shape domain-specific learning strategies to fit adaptive contingencies (Boyd \& Richerson, 1985; Gallistel, 1990, 1995; Lumsden \& Wilson, 1981; Pulliam \& Dunford, 1980; Rozin, 1976; Tooby \& Cosmides, 1992).

\section{When is Learning Adaptive?}

There is some optimum degree and form of plasticity for a given organism inhabiting a given range of environments (see Scheiner 1993). If the environ- 
ment is uniform and constant, then it is adaptive for the population to fix innately the behavior that arose through ontogenetic plasticity: Complete genetic assimilation avoids the considerable costs of the time it takes to learn the behavior, the costs of errors along the way, and the costs of not learning the behavior at all under some conditions (see, e.g., Boyd \& Richerson, 1985; Scheiner, 1993; Wcislo, 1989). The organism can adapt to temporal and spatial heterogeneity in the environment in two ways. The genotypes of all living things specify contingent responses determined by genotypically delineated cues. A seed germinates only under certain conditions, then sends its roots in the direction of gravitational attraction while its leaves grow upward. Many organisms reproduce asexually under optimal conditions and sexually under more adverse conditions. In these cases, the organism's behavior is plastic but not learned: The function that relates environmental conditions to behavior is predetermined by the genotype. An organism can evolve genetically predetermined responses to any number of specified environmental conditions, making its phenotype adaptively responsive to diverse environments. This does not require learning and indeed, if a given environmental cue reliably predicts a given optimal response, it is a liability for the organism to have to discover the correlation for itself in its own lifetime experience. If a given cue predicts the same adaptively relevant condition in most environments that an organism encounters, then natural selection should tend to produce rigid genotypic determination of the function relating cue to response. However, as phenotypic plasticity decreases, the range of niches to which the organism can adapt narrows and the speed of its reaction to environmental changes slows.

Learning is adaptive and should be selected if there is variability in the patterns of co-occurrence of cues and optimally adaptive responses, or if a limited set of fixed responses is less advantageous than the generation of an (indefinitely) abundant set of constructed responses based on an effective strategy. So, for example, if a smile on the face of a male adult stranger toward an isolated female adult always predicts friendly behavior that the perceiver should respond to with trusting affiliation, then natural selection will tend to hardwire this response into the mind. If, however, male smiles can signal many different meanings that cannot be predicted without local experience, then it is adaptive to learn from experience, imitation, or instruction. Learning always has a cost and is selected to the extent that the experience of the population over many generations cannot be effectively used to predict the adaptive response.

Learning is also adaptive when the necessary information is reliably available in the environment and readily accessible by a learning mechanism that can evolve more easily than a genetic mechanism for storing the same information. This is probably part of the reason that the lexicons of human languages are not innate. (Another reason must be that it is highly adaptive to be able to learn new words for referring to new things and performing new speech acts.)

Learning is only possible when the organism has a genotypically structured strategy that enables it to (a) focus attention on potentially relevant cues, (b) evaluate plausible functions connecting these cues to a repertoire of potentially appropriate responses, and (c) effectively deploy organized adaptive actions. This was Chomsky's $(1959,1988)$ message when he argued for the existence of a language acquisition device; Seligman (1970) when he argued for functional preparedness to learn in certain ways; Rozin (1976; Rozin \& Kalat, 1972) when he showed that there must be adaptive specializations for learning; Garcia (Garcia \& Ervin, 1968) when he demonstrated specialized capacities to make associations relevant to the animal's niche; Boyd and Richerson (1985) when they analyzed the conditions selecting, respectively, for individual learning, cultural inheritance, or genetic inheritance of behavior; and Tooby and Cosmides (1992) when they posited adaptive cognitive modules (see also Hinde \& Stevenson-Hinde, 1973). Pure unstructured plasticity that calculated all correlations among all features of the environment and randomly explored all possible responses would not be adaptive: It would only very slowly and rarely arrive at adaptive responses. In any event, such a mechanism would be computationally impossible.

Using this foundation, consider how humans could have evolved mods that depend on cultural complements.

\section{The Human Adaptations for Social Cumulation, Complementation, and Generativity}

Often the evolution of a new adaptation results in a change in selection that leads to the evolution of other adaptations, which in turn produce selection for the evolution of further adaptations in a process of "sequential evolution" (Seaborg, 1999). Sometimes the second- or third-order adaptations have strong effects on the selection that shapes the first adaptation, resulting in evolutionary feedback. This kind of sequential evolution and evolutionary feedback can result in the relatively sudden and dramatic adaptive shifts that appear as punctuated evolutionary change (Seaborg, 1999). Complementarity theory posits a process of sequential evolution with feedback that led to the cultur- 
ally organized eusociality of Homo sapiens (cf. Campbell, 1983, for another account).

\section{Cumulation}

Humans are characterized by three connected adaptations: (a) socially transmitted knowledge and therefore cumulative learning, (b) extremely complex social coordination, and (c) highly varied social systems that are adapted to specific ecologies and social environments. Taken together, these three adaptations are called culture, but it is analytically useful to separate them. Consider first the importance of socially transmitted skills that accumulate across generations. As previously noted, humans have evolved a set of uniquely powerful capacities for transmitting knowledge and skills; the most important of these capacities is the collection of skills called imitation (see Fiske, 1999). In addition, the social construction of spaces and artifacts facilitates the transmission of knowledge and practices. Tools, especially, guide action and are a powerful medium of social inheritance in conjunction with imitation. Humans have language and other symbol systems, permitting them to store all kinds of knowledge and competence in propositional and narrative form. In addition, humans often issue commands to younger or subordinate persons that more or less incidentally permit the latter to learn new skills. In some cultures people sometimes instruct others. These adaptations must have coevolved with the long dependency and delayed sexual maturation that are distinctive of Homo.

Once humans began to be able to learn from each other, the accumulating knowledge and skills available would have placed a tremendous premium on the capacity to learn, remember, and apply such capacities. Each individual would have had the opportunity to acquire and utilize far more skills than any individual could ever acquire on its own in one lifetime. In principle, the knowledge and skills available to any individual could grow exponentially if each individual could learn from several ancestors and peers. Special cognitive capacities would be needed to store such a vast body of knowledge and use such a vast array of skills. In this context, those individuals who exhibited the best social learning would have a tremendous advantage, leading to a very intense selection for the genotypic proclivity to learn from others. The more varied the environmental contingencies and the greater the cost of individual learning, the greater the benefits of capacities for learning socially transmitted knowledge (Boyd \& Richerson, 1985). The value of such learning would be enhanced if the most competent learners could parlay their knowledge into social sta- tus, offering access to their knowledge in return for other benefits.

Humans have uniquely powerful capacities for learning from others, in conjunction with ancillary memory and performance capacities (cf. Donald, 1991). To distinguish this adaptation from individual learning, let us call it cumulation. (Other terms, such as social learning, have also been used in somewhat different theoretical frameworks.) Note that cumulation does not require any high degree of social cooperation beyond that which is involved in merely permitting others to observe and imitate. Capacities for cumulation must have evolved gradually from simpler observational capacities that modern apes currently possess (Tomasello \& Call, 1997; Whiten \& Custance, 1996). A recent analysis of the variability of behavior of seven chimpanzee groups suggests that Pan troglodytes apparently has some capacity for cumulation. Whiten et al. (1999) identified 39 socially transmitted behaviors that do not appear to be explained by individual learning in response to differing ecologies. Most of these involve ways of using tools. My interpretation is that these cumulated practices may be transmitted by stimulus enhancement rather than sophisticated functional imitation. (That is, observation facilitates associations that provide shortcuts to learning: Once chimpanzees observe an association between a tool and a reward, they are capable of exploring individually and eventually learning for themselves how to use the tool to get the reward.)

\section{Complementation}

Cumulation is the capacity to learn what others have learned (e.g., how to build a fire, cook, make a tool, track an animal, or find tubers), but what is learned by cumulation consists of skills that can be used alone, without further interaction with the people from whom one has learned. Building on this imitative capacity, humans evolved a step further: the capacity to learn coordination devices from each other. Among toddlers, imitating another person is often the first step in developing an interaction with them (Eckerman \& Didow, 1996). One hypothetical process leads to a communal sharing relationship: Imitating another person results in a sense of similarity (i.e., imitation becomes conformity and identification). Another process leads to equality matching via turn taking (e.g., he does it; her imitation of him becomes taking a turn), matching (she does it, he matches her action; she takes one and then, by his taking one for himself, he keeps even), or in-kind reciprocation (A hits B and then B hits A, or A helps B and then B helps A). Similarly, ritual could evolve from a repetitive reflection of each other's actions: 
Imitation becomes reenactment. Indeed, people performing rituals often explain their action as the reproduction of previous performances: "It is what we found when we were born and what we leave behind when we die," say the Moose.

Thus, it is a smooth but significant step to go from learning a technique to coordinating an interaction. This adaptive step may build on the fact that imitating another person's action potentially leads to understanding their point of view. Suppose A takes something from $\mathrm{B}$, then $\mathrm{B}$ takes something from $\mathrm{A}$; now each may begin to understand the other's perspective. In human children, we see this kind of imitation: role playing in which the child plays the part of the parent (i.e., from the point of view of the parent). When the child does this kind of role playing, the child may act as mother while a doll or sibling occupies the role that the child normally plays.

We call this adaptation complementation; it entails cumulation but in addition consists of the capacity to use the acquired device to mediate a coordinated interaction. What is learned is not simply knowledge or a skill-it is a device for constructing action that complements the action of the people from whom one has learned. Complementation is learning from another person a model, schema, or artifact that they are using to organize their action; this model or artifact then mediates the mutual construction and interpretation of each other's action.

Coordinated social interaction can be achieved by genetic adaptation to the social actions of other members of the species, as social insects do, without complementation (Wilson, 1974). Among close kin, selection can facilitate coordination when the behavior of all is genotypically fixed: Social insects can coadapt to relatively unchanging, invariant, hence predictable behavior by others in the colony. In this case, the cognitive structures used to produce an action may be entirely distinct from those used to respond to it. Nonhuman primates apparently lack much sense of what others are thinking: They do not have a subtle theory of mind, if they have any at all. They may coordinate interaction using a set of social coadaptations in which the cognitive structures for constructing behavior are distinct from those used to respond to others, or they may use simple shared models (Haslam, 1997). Social coordination through genetically fixed behavior eliminates the difficult learning problem that complementation poses for the individual, eliminating the costs of delays, initial errors, and failures to learn in the face of novel conditions. Strategies for independent individual learning of social skills permit some coordination as well, but only to a limited extent. Neither direct genotypic control nor individual learning permit complex, flexible complementarity. Complementation makes the learn- ing and mutual adjustment problems very difficult, but if natural selection solves these problems, complementation permits individuals to vary their forms of coordination to adapt to local conditions. It also permits rapid collective changes from one coordination device to another.

\section{Generativity}

Complementation is the capacity to learn coordination devices one by one. For example, at this evolutionary stage, the individual can observe others and learn to share meat or learn from others how to take turns using a tool to dig for tubers. Complementation can develop into something further if the coordination devices can be generalized to other situations by freeing them of their specific content and context. Complementation is the adaptation that enables individuals to learn to share meat, but at this stage learning to share meat does not enable the learner to share responsibility for providing the meat or to make a decision by consensus. Complementation enables the individual to observe, imitate, and learn to take turns using a digging tool, but having learned this the individual still does not know how to take turns cooking, how to exchange a tuber received today for a tuber returned tomorrow, or how to conduct a fair lottery. This is the next step.

This adaptive step gradually results from natural selection for proclivities to learn coordination devices. Certain kinds of coordination devices are versatile and effective: They permit complementation that offers adaptive advantages to participants coordinating an infinite variety of contextually diverse interactions. Relational models, for one example, are structures that have exceptionally powerful fundamental properties. These structures remain the same under important types of transformation (a property called uniqueness in measurement theory) and every element has the same relational properties (homogeneity; see Fiske, 1991, p. 229). This makes these four structures effective for organizing almost any social activity.

Consequently, natural selection would favor capacities to generalize such coordination devices beyond the context in which they were initially learned. For example, an individual would benefit greatly if, after learning the basic structure and operations of an ordered Abelian group in the form of turn taking, it subsequently developed any capacity to use ordered Abelian groups for balanced reciprocity or evenly dividing tasks (Fiske, 1991, pp. 207-223). This assumes, of course, that people associated preferentially with close kin likely to share the same mutations. Similarly, an individual who imitated others and learned to use 
equivalence relations to share shellfish would benefit greatly from any proclivity to apply equivalence relations to sharing pine nuts or taking joint responsibility for tending the fire. In general, an increase in fitness would be conferred by any mutation that facilitated the invention of new uses for a relational model; reliable, rapid learning of a relational model; or proficient use of one. By small increments, Homo sapiens must have acquired greater and greater faculties for learning to use relational models. In much the same manner, natural selection would steadily increase proclivities to learn and use other valuable coordination devices. These devices were devised by processes of individual learning and transmitted initially by complementation capacities such as imitation. Once this social transmission was pervasive and enduring, the coordination devices become part of the environment to which Homo adapted genetically in subsequent generations.

By the logic we reviewed previously in the section on natural selection for ontogenetic strategies, this process of natural selection should continue up to the point at which further genetic assimilation reduces adaptively advantageous versatility, flexibility, and capacity to change quickly in response to environmental changes and new opportunities. Furthermore, natural selection should construct a limited number of proclivities that each facilitate the widest functionally possible range of most beneficial coordination devices. This led by small increments to the evolution of proclivities that consisted of those structures specifying all of the relations and operations that are functionally valuable in diverse contexts for a wide variety of adaptive coordinations. However, these structures would become liabilities if they evolved beyond this point and began to be specialized for particular, limited contexts in ways that interfered with a range of numerous other potentially more beneficial uses. In short, what evolved were cognitive developmental proclivities innately defining coordination structures and processes of wide adaptive value but leaving open the specification of how, when, with whom, and with respect to what aspects of social life they are used. In other words, Homo sapiens evolved generative mods requiring preos.

Mods linked with preos are generative at approximately three levels: using a mod with a set of preos generates a social institution (e.g., marriage); further precision in specifying preos, or additional preos, generates a particular relationship (e.g., my own marriage); and applying these preos to particular circumstances generates specific interactive events (I bathe the children tonight). In language, this is evident in the variety of languages purportedly generated by universal grammar, the variety of dialects and styles within each language, and the innumerable utterances that a speaker can produce and comprehend. This generativity makes human sociality uniquely adaptive in two respects.

First, people within a community or interaction network can use the same mod with different preos to generate innumerable cultural institutions, particular relationships, and specific interactions (Fiske, 1991). Thus, equality matching can be used to make group decisions (e.g., by voting), to organize exchange (e.g., by even, in-kind reciprocation), to structure violence (an eye for an eye, a tooth for a tooth), to make moral judgments (equal treatment and equal opportunity), to divide work (take turns), and so forth, indefinitely. Using it to organize exchange, we can construct rotating credit associations in which participants meet weekly, each bringing $\$ 100$ and one member taking home the entire pot each week. We can conduct exchanges in which you invite me to dinner and I owe you a dinner in return. We can exchange baby-sitting in a cooperative in which the medium is coupons. Your baby-sitting coop may count each hour for each child as one coupon, while my coop exchanges a night out for another night out, regardless of the number of hours and children. Thus, a very small set of mods permit people to easily coordinate innumerable activities with mutual understanding: All you have to do is perceive what mod is being used with what preos.

Second, this kind of generativity makes it possible for people to use the same CCDs to generate highly varied behavior in different circumstances: Different cultures can generate different systems of marriage in different ecological and political-historical contexts; different dyads can generate marriages appropriate to their pair of personalities and their social circumstances; dyads or groups can generate distinct actions that take into account the immediate situation. So in one culture, marriage is governed primarily by communal sharing, in another by authority ranking, by equality matching in a third culture, and market pricing in a fourth. If equality matching is the prescriptive CCD for marriage in one culture, that still leaves room to decide whether to take turns cooking dinner alternate nights or to split the cost of eating out. If a marriage is mediated by equality matching implemented as cooking alternate nights, one still has lots of room for interpretation, improvisation (and perhaps conflict) each night over what constitutes "cooking dinner" (e.g., ordering pizza), and who should cook the night after a dinner party. This makes a few generative mods capable of adapting to all kinds of conditions. In effect, the process is recursive: The conjunction of a mod and a preo generates a CCD that operates globally to define a system, but that CCD must be applied with further preos to define a specific relationship and with still more preos to construct each particular interaction. 
This is generativity: the capacity to construct social coordination in indefinite ways using a finite set of mods (concatenated and nested) with an indefinite set of socially transmitted, shared preos. Humans gain enormous fitness advantages through the evolution of mods and the social transmission of complementary preos that enable them to generate indefinitely many implementations of the same model-implementations that are unique but coordinated with sets of local partners. Humans are culturally social animals whose fitness depends on coordinating in culturally and situationally varied ways: There is no an advantage in being able to speak a language if it is not the language the other people around you speak. Nor is there any advantage in constructing novel utterances if the listeners do not understand them. Generativity is the extraordinarily beneficial adaptive capacity to combine productivity and creativity with social coordination based on complementarity. Generativity results from combining genotypic inheritance of universal mods with social transmission, local diffusion, ad hoc negotiation, and extemporization of preos. Mods and preos complete each other, permitting complementarity of action in indefinitely many, varied, and novel institutions, relationships, and interactions.

Natural selection for open-ended-hence generative-mods is also the result of the quantitative limitations of the information that the genome can encode directly compared to the enormous capacity of the brain and its cognitive processes. The genome can encode the design for a brain that can learn far more than the genome can encode directly. For example, the available portion of the genome cannot encode the lexicon of a human language, knowledge of all social arrangements, or competence with all technologies that can be adaptive under all environmental conditions. However, the genome can encode mods for assimilating cumulated local preos and then generating any human lexicon, technology, or social system.

A third factor favoring natural selection of generative mods coupled with socially transmitted preos is speed of adaptive response. Creative invention coupled with social transmission result in cultural evolution that is much more rapid than genetic evolution (Boyd \& Richerson, 1985). Baldwinian adaptation cannot track and genetically assimilate cultural inventions whenever they are adaptive fast enough to keep up with cultural evolution. This is approximately the temporally extended equivalent of cultural diversity at one point in time: Just as genetic evolution within one gene pool cannot assimilate the diversity of cultural inventions in different groups and networks, so the genotype cannot respond very rapidly with fixed action patterns to adaptive opportunities and hazards (see Boyd \& Richerson, 1995). Hence, there is strong selec- tion to evolve mods that permit rapid transition to new CCDs in response to environmental changes-including novel cultural inventions.

A fourth aspect of generativity is the ability to combine CCDs to construct complex forms of social coordination. The same CCD can be nested or concatenated with itself recursively, or people can link and nest multiple CCDs. For example, a group decision can be made by consensus (communal sharing) that a king should be elected (equality matching) to rule arbitrarily over us (authority ranking) because it is the most efficient, cost-effective system of government (market pricing). This is a basic principle of syntax but it operates in other forms of generative social complementation as well.

Cumulation, complementation, and generativity are synergistic adaptations: The adaptive advantages of each are greatly amplified when linked to the others. In addition, each of these three adaptations must have been greatly facilitated by and in turn provided opportunities for further adaptations based on inclusive fitness (Frank, 1998; Hamilton, 1964) and reciprocal altruism (Trivers, 1971). Both inclusive fitness and reciprocal altruism are functions of the ratio of benefits conferred to another divided by the costs to the actor. Cumulation, complementation, and generative CCDs provide uniquely powerful opportunities for conferring major benefits at little cost to the donor. Consequently, natural selection for adaptations based on the mechanisms of inclusive fitness and reciprocal altruism greatly enhances and in turn is greatly enhanced by the adaptive value of cumulation, complementation, and generative CCDs. Thus, they mutually facilitate each other's evolution. (I shall defer more detailed discussion of this complementarity to a subsequent publication.)

\section{Symbiosis Between Mods and Preos}

The complementarity of the reproductive processes of mods and preos can readily result in a kind of symbiosis between them. To reproduce, the mods need the social transmission of compatible preos that result in the construction of adaptive CCDs. Conversely, the preos and the cultural coordination devices based on them need compatible mods; the only niche in which preos can reproduce is a social system comprised of humans with congruent mods. Thus, mods may often evolve so as to support as effectively as possible the most extensive social transmission and greatest expression of the largest number of genotypically adaptive congruent cultural paradigms. This does not mean total plasticity: The mod is only adaptive to the extent that it channels the generation of cultural paradigms in fitness-enhancing directions. It does mean, however, that a mod that originally 
evolved because it facilitated one type of congruent cultural construct may further evolve to more effectively facilitate diverse adaptive expressions of that mod and to facilitate many others. That is, the mod evolves to ensure the reliable social transmission of any preos that are necessary or sufficient to translate the mod into adaptive CCDs. Conversely, cultural paradigms reproduce most prolifically when the population contains the greatest number of humans with the necessary potentiating mods. Hence, cultural paradigms should gradually transform-evolve-to maximally enhance the inclusive genotypic fitness of the mods that facilitate the social reproduction of those paradigms.

However, this mutual enhancement is a function of the symbiosis of a mod with a preo; it obtains to the extent that the reproduction of either depends entirely on the other. Preos will tend to transform so as to become congruent with mods other than their original complements: This makes such paradigms multiply potentiated. To the extent that a preo comes to depend on the conjunction of a set of mods, requiring Mod A, Mod B, and Mod C (etc.), this will limit the further transformations that it can undergo because few transformations will retain congruence with all the potentiating mods. This kind of coevolutionary feedback may lead to adaptive stasis (Seaborg, 1999). However, if a preo becomes transformed so that it is congruent with Mod A, Mod B, or Mod C (etc.), then it can be socially reproduced independently of any one of these mods. This makes the preo's reproductive potential partially independent of any one mod and hence its interests diverge from each one. The preo can therefore become less symbiotic and more parasitic, exploiting a mod in ways that are not necessarily entirely conducive to the fitness of the mod.

Conversely, a mod will tend to evolve so as to facilitate the production of additional adaptive cultural paradigms beyond the one in conjunction with which it originally evolved. The greater the number and the greater the adaptive value of the cultural paradigms that any one mod facilitates, the greater the fitness of the mod. However, once again, a mod that evolves to potentiate multiple cultural paradigms will become constrained in ways that limit its further evolution: Subsequent modifications will be less likely to be congruent with all of the cultural paradigms that it already supports. A mod that is congruent with multiple adaptive cultural paradigms no longer depends exclusively on any one of them, and hence natural selection of this mod will become less closely linked to its potentiation of each one of the congruent constructs. This weakens the mod's symbiotic support of the reproductive interests of any one of its congruent cultural paradigms.

Natural selection operates on culturally mediated phenotypes (cf. Caporael, 1997). On the scale of one life span-for the individual-it is adaptive to coordinate according to local cultural norms, to participate in the accessible communities and networks, regardless of whether the local CCDs are optimal in the abstract. An individual is at a great disadvantage if he or she is the only speaker of a language that would be optimal if everyone spoke it. Similarly, there are great costs to the sole person who shares generously-despite the fact that it may be beneficial to all participants if only they all conducted their transactions in such a manner. Humans depend fundamentally on their social relationships for most of their basic needs and, therefore, must generally conform to most of the important CCDs that prevail in their own communities and networks. This means that humans must be tuned to their own particular cultures. They must be adept at learning the particular CCDs through which their own culture implements, elaborates, transforms, and connects the generic human potentials. Meanwhile, over the long run, natural selection shapes the psyche to be capable of learning and motivated to utilize the full cultural diversity of CCDs that have been adaptive in the population. This means that natural selection slowly tracks the historical assortment of adaptive cultural inventions. On the other side, over the long run, the kinds of cultural innovations that resonate most strongly with evolved psychological proclivities will most readily diffuse and persist. So culture adapts to human mods. Overall, these capacities facilitate new kinds of adaptation, including the possibility of a single gene poolHomo sapiens - developing very diverse, highly specialized, yet socially coordinated, adaptations to specific local conditions along with rapid responses to environmental changes.

\section{Conclusions}

The theory outlined in this article suggests that cultural transmission; social coordination; cognitive, emotional, and developmental psychology; and natural selection are so highly interdependent in Homo sapiens that they cannot be understood separately. These systems have become complementary, and each requires the others to complete it. Natural selection takes advantage of cultural innovations, and culture capitalizes on evolved potentials. The psyche exploits cultural tools, and social relations build on culturally constituted implementations of evolved psychological proclivities. It is not that human cultural transmission, social processes, human psychology, and natural selection influence each other. They rely on each other, presuppose each other, operate by virtue of each other, and are functionally incomplete without each other. The human psyche has evolved to 
function as a cultural psyche, dependent on cultural precedents, prototypes, and paradigms that make social coordination possible. Conversely, human cultures have adapted themselves to the evolved psychological proclivities that reproduce them. Human nature is cultural and humans are cultural by nature.

\section{Conjoining Preos and Mods to Construct CCDs}

Complementarity theory suggests that there are a number of important cultural coordinating devices that require specific evolved psychological proclivities. These cultural coordinating devices are essential for meaningful complementarity in human sociality: They are necessary for the construction and conduct of human social practices, relationships, organized groups, institutions, and societies. These CCDs mediate communicative action, permitting people to generate actions that others can anticipate and understand. People must use one or more CCDs to jointly construct social practices that consist of coordination in which the actions of each person presuppose and make sense only with reference to complementary actions of other participants. The cultural reproduction and systematic transformation of CCDs is made possible because humans have evolved corresponding mods that dispose them to learn and interact in certain specific ways. Conversely, mods evolve when people devise, share, and transmit preos for adaptive CCDs. In this sense, mods are products of a distinctive culturally potentiated sequence of adaptation: first, cumulation of skills across generations, then complementation of actions, and finally, generativity of CCDs. Thus, cultural coordination devices such as language, relational models, rituals, moral interpretations of misfortune, taboos, and religion are the product of evolved, universal psychological proclivities conjoined with socially transmitted, culture-specific complements.

\section{Implications For Social Psychological Research}

Complementarity theory implies that researchers analyzing social psychological processes should consider how these processes

- Have shaped natural selection and culture;

- Are potentiated and channeled by evolved proclivities;

- Are developed by inherently sociable children because they desire to relate;
- Are learned by very structured processes; and

- Are intrinsically (not incidentally) variable across cultures.

Complementarity theory reminds social psychologists that culture is not an exogenous variable: The generativity that results in cultural diversity is built into many basic developmental, cognitive, and social psychological processes. Complementarity theory highlights the idea that humans generally do not think about each other primarily as objects with features: People do not focus on others' individual attributes. The crux of social psychology is the coordination of motives, emotions, evaluations, cognitions, and actions. People organize their action in ways that are complementary to the actions of their partners, opponents, and observers in social relationships and groups. When people think about each other they may ask, "What kind of person is this?" However, they ask this question with reference to the possibilities the person offers-and the problems the person poses-for coordinated interaction: for communication, for relationships, and for participating in potentially complementary roles. People consider each other in terms of the congruity or incongruity of their motives, emotions, evaluations, cognitions, and actions.

Complementarity theory focuses on the adaptive specializations that characterize us as eusocial, ultracultural, culturally social animals. Our fitness and our everyday well-being depend on our culturally organized sociality. That sociality is not based primarily on general purpose cognitive processes. It is not based on rigid fixed action patterns that dictate identical genotypically controlled social behavior that is invariant in every community around the world. Nor is our sociality the product of incomparable, arbitrary cultural constructions generated idiosyncratically in each community or each interaction. Humans are social beings who have evolved a finite set of psychological proclivities that are capable of being completed by an infinite number of congruent socially transmitted prototypes and principles, enabling us to construct devices to coordinate with each other. Consequently, we can coordinate in innumerable mutually intelligible, mutually meaningful, culturally distinct yet universally structured institutions, particular relationships, and specific social acts.

\section{References}

Atran, S. (1998). Folk biology and the anthropology of science: Cognitive universals and cultural particulars. Behavioral and Brain Sciences, 21, 547-609. 
Baldwin, J. M. (1896). A new factor in evolution. The American Naturalist, 30, 441-451, 536-553.

Boyd, R., \& Richerson, P. J. (1985). Culture and the evolutionary process. Chicago: University of Chicago Press.

Boyd, R., \& Richerson, P. J. (1989). The role of evolved predispositions in cultural evolution. Ethology and Sociobiology, 10, 195-219.

Boyd, R., \& Richerson, P. J. (1995). Why does culture increase human adaptability? Ethology and Sociobiology, 16, 125-143.

Boyer, P. (1994). The naturalness of religious ideas: A cognitive theory of religion. Berkeley: University of California Press.

Buss, D. M. (1994). The evolution of desire: Strategies of human mating. New York: Basic Books.

Campbell, D. T. (1983). Two distinct routes beyond kin selection to ultrasociality: Implications for the humanities and social sciences. In D. Bridgeman (Ed.), The nature of prosocial development: Theories and strategies (pp. 11-41). New York: Academic.

Caporael, L. (1997). The evolution of truly social cognition: The core configurations model. Personality and Social Psychology Review, 1, 276-298.

Cavalli-Sforza, L. L., \& Feldman, M. W. (1981). Cultural transmission and evolution: A quantitative approach. Princeton, NJ: Princeton University Press.

Chomsky, N. (1959). A review of B. F. Skinner's "Verbal Behavior." Language, 35, 26-58.

Chomsky, N. (1988). Language and problems of knowledge: The Managua lectures. Cambridge, MA: MIT Press.

D’Andrade, R. G., \& Strauss, C. (Eds.). (1992). Human motives and cultural models. New York: Cambridge University Press.

Deacon, T. W. (1998). The symbolic species: The co-evolution of language and the brain. New York: Norton.

Donald, M. (1991) Origins of the modern mind: Three stages in the evolution of culture and cognition. Cambridge, MA: Harvard University Press.

Dulaney, S., \& Fiske, A. P. (1994). Cultural rituals and obsessive-compulsive disorder: Is there a common psychological mechanism? Ethos, 22, 243-283.

Durham, W. H. (1991). Coevolution: Genes, culture, and human diversity. Stanford, CA: Stanford University Press.

Eckerman, C. O., \& Didow, S. M. (1996). Nonverbal imitation and toddlers' mastery of verbal means of achieving coordinated action. Developmental Psychology 32, 141-152.

Elman J. L., Bates, E. A., \& Johnson, M. H. (1996). Rethinking innateness: A connectionist perspective on development. Cambridge, MA: MIT Press.

Fiske, A. P. (1991). Structures of social life: The four elementary forms of human relations. New York: Free Press (Macmillan).

Fiske, A. P. (1992). The four elementary forms of sociality: Framework for a unified theory of social relations. Psychological Review, 99, 689-723.

Fiske, A. P. (1998). Human sociality. International Society for the Study of Personal Relationships Bulletin, 14(2), 4-9.

Fiske, A. P. (1999). Learning culture the way informants do: Observation, imitation, and participation. Unpublished manuscript.

Fiske, A. P. (2000). Taboo. Manuscript in preparation.

Fiske, A. P., \& Haslam, N. (1996). Social cognition is thinking about relationships. Current Directions in Psychological Science, 5, 143-148

Fiske, A. P. , \& Haslam, N. (1997). Is obsessive-compulsive disorder a pathology of the human disposition to perform socially meaningful rituals? Evidence of similar content. Journal of Nervous and Mental Disease, 185, 211-222.

Fodor, J. A. (1983). The modularity of mind. Cambridge, MA: MIT Press.

Frank, S. A. (1998). Foundations of social evolution. Princeton, NJ: Princeton University Press.
Gallistel, C. R. (1990). The organization of learning. Cambridge, MA: MIT Press.

Gallistel, C. R. (1995). The replacement of general-purpose theories with adaptive specializations. In M. S. Gazzaniga (Ed.), The cognitive neurosciences (pp. 1255-1267). Cambridge, MA: MIT Press.

Garcia, J., \& Ervin, F. R. (1968). Gustatory-visceral and telereceptor-cutaneous conditioning-Adaptation to internal and external milieus. Communications in Behavioral Biology, A1, 389-415.

Greenfield, P. M., Maynard, A. E., Boehm, C., \& Yut-Schmidtling, E. (in press). Cultural apprenticeship and cultural change: Tool learning and imitation in chimpanzees and humans. In S. T. Parker, J. Langer, \& M. L. McKinney (Eds.), The evolution of behavioral ontogeny. Santa Fe, NM: School of American Research Press.

Hamilton, W. D. (1964). The genetical evolution of social behavior. Journal of Theoretical Biology, 7, 1-52.

Haslam, N. (1997). Four grammars for primate social relations. In J. Simpson \& D. Kenrick (Eds.), Evolutionary social psychology (pp. 293-312). Mahwah, NJ: Lawrence Erlbaum Associates, Inc.

Hinde, R. A., \& Stevenson-Hinde, J. (Eds.). (1973). Constraints on learning: Limitations and predispositions. New York: Academic.

Hinton, G. E., \& Nowlan, S. J. (1987). How learning can guide evolution. Complex Systems, 1, 495-502.

Hirschfeld, L. A., \& Gelman, S. A. (Eds.). (1994). Mapping the mind: Domain specificity in cognition and culture. New York: Cambridge University Press.

Laland, K. N., Richerson, P. J., \& Boyd, R. (1993). Animal social learning: Toward a new theoretical approach. Perspectives in Ethology, 10, 249-277.

Lancy, D. F. (1996). Playing on the mother-ground: Cultural routines for children's development. New York: Guilford.

Lave, J., \& Wenger, E. (1991). Situated learning: Legitimate peripheral participation. Cambridge, England: Cambridge University Press.

Lumsden, C. J., \& Wilson, E. O. (1981). Genes, mind, and culture: The coevolutionary process. Cambridge, MA: Harvard University Press.

Mayley, G. (1997). Landscapes, learning costs, and genetic assimilation. Evolutionary Computation, 4, 213-234.

Morgan, C. L. (1896). On modification and variation. Science, 4, 733-740.

Nagel, K., Olguin, R. S., \& Tomasello, M. (1993) Processes of social learning in the tool use of chimpanzees (Pan troglodytes) and human children (Homo sapiens). Journal of Comparative Psychology, 107, 174-186.

Osborn, H. F. (1896a). A mode of evolution requiring neither natural selection nor the inheritance of acquired characters. Transactions of the New York Academy of Science, 15, 141-142, 148.

Osborn, H. F. (1896b). Ontogenetic and phylogenetic variation. Science, 4, 786-789.

Pulliam, H. R., \& Dunford, C. (1980). Programmed to learn: An essay on the evolution of culture. New York: Columbia University Press.

Rogoff, B., Baker-Sennett, J., Lacasa, P., \& Goldsmith D. (1995). Development through participation in sociocultural activity. In J. J. Goodenow, P. Miller, \& F. Kessel (Eds.), Cultural practices as contexts for development (pp. 45-66). San Francisco: Jossey-Bass.

Rozin, P. (1976). The evolution of intelligence and access to the cognitive unconscious. In J. M. Sprague \& A. E. Epstein (Eds.), Progress in psychobiology and physiological psychology, 6, 245-280. 
Rozin, P. (1982). Human food selection: The interaction of biology, culture, and individual experience. In L. M. Barker (Ed.), The psychobiology of human food selection (pp. 225-254) Westport, CT: AVI Publishing.

Rozin, P., \& Kalat, J. (1972). Learning as a situation-specific adaptation. In M. E. P. Seligman \& J. Hager (Eds.), The biological boundaries of learning (pp. 66-97). New York: Appleton-Century-Crofts.

Sahlins, M. (1972). Stone age economics. New York: Aldine.

Scheiner, S. M. (1993). Genetics and evolution of phenotypic plasticity. Annual Review of Ecology and Systematics, 24, 35-68.

Seaborg, D. M. (1999). Evolutionary feedback: A new mechanism for stasis and punctuated evolutionary change based on integration of the organism. Journal of Theorertical Biology, 198, $1-26$.

Seligman, M. E. P. (1970). On the generality of the laws of learning. Psychological Review, 77, 406-418.

Sperber, D. (1996). Anthropology and psychology: Towards an epidemiology of representations. In D. Sperber (Eds.), Explaining culture: A naturalistic approach (pp. 56-76). Oxford, England: Blackwell.

Tomasello, M. (Ed.). (1998). The new psychology of language: Cognitive and functional approaches to language structure. Mahwah, NJ: Lawrence Erlbaum Associates, Inc.

Tomasello, M., \& Call, J. (1997). Primate cognition. New York: Oxford University Press.
Tomasello, M., Kruger, A. C., \& Ratner, H. H. (1993). Cultural learning (with commentaries and reply). Behavioral and Brain Sciences, 16, 495-552.

Tooby, J., \& Cosmides, L. (1992). Psychological foundations of culture. In J. H. Barkow, L. Cosmides, \& J. Tooby (Eds.), The adapted mind: Evolutionary psychology and the generation of culture (pp. 19-36). New York: Oxford University Press.

Trevarthen, C. (1988). Universal co-operative motives: How infants begin to know the language and culture of their parents. In G. Jahoda \& I. M. Lewis (Eds.), Acquiring culture: Cross-cultural studies in child development (pp.37-91). London: Croom Helm.

Trivers, R. L. (1971). The evolution of reciprocal altruism. The Quarterly Review of Biology, 46, 35-57.

Waddington, C. (1975). The evolution of an evolutionist. Edinburgh, Scotland: Edinburgh University Press.

Wcislo, W. T. (1989). Behavioral environments and evolutionary change. Annual Review of Ecology and Systematics, 20, 137-169.

Whiten, A., \& Custance, D. (1996). Studies of imitation in chimpanzees and children. In C. M. Heyes \& B. G. Galef, Jr. (Eds.), Social learning in animals: The roots of culture (pp. 291-318). San Diego: Academic.

Whiten, A., Goodall, J., McGrew, W. C., Nishida, T., Reynolds, V., Sugiyama, Y., Tutin, C. E. G., Wrangham, R. W., \& Boesch, C. (1999). Cultures in chimpanzees. Nature, 399, 682-685.

Wilson, E. O. (1974). The insect societies. Cambridge, MA: Harvard University Press. 
Copyright of Personality \& Social Psychology Review is the property of Lawrence Erlbaum Associates and its content may not be copied or emailed to multiple sites or posted to a listserv without the copyright holder's express written permission. However, users may print, download, or email articles for individual use. 